DOI: 10.1136/annrheumdis-2018-eular.6514

SATURDAY, 16 JUNE 2018

How do you sleep?

\section{OP0361-HPR SLEEPING PROBLEMS AND ANXIETY IS ASSOCIATED TO CHRONIC MULTISITE MUSCULOSKELETAL PAIN IN} SWEDISH HIGH SCHOOL STUDENTS

J.S. Malmborg ${ }^{1}$, A. Bremander ${ }^{1}$, M.C. Olsson ${ }^{1}$, A.-C. Bergman², S. Brorsson², S. Bergman ${ }^{3} .{ }^{1}$ The Rydberg Laboratory for Applied Sciences, Halmstad University; ${ }^{2}$ Spenshult Research and Development Center, Halmstad; ${ }^{3}$ Primary Health Care Unit, Department of Public Health and Community Medicine, Institute of Medicine, The Sahlgrenska Academy, University of Gothenburg, Gothenburg, Sweden

Background: The relationship between chronic multisite musculoskeletal pain (CMP) and sleep is complex, where pain can lead to sleeping problems and lack of sleep can intensify the pain perception. Most previous studies relates to adults, but adolescents may also suffer from CMP, and there is a need for more knowledge regarding the relationships between CMP and sleeping problems, stress, anxiety, depression, and health status.

Objectives: To study background factors associated to CMP in first year Swedish high school students.

Methods: First year Swedish high school students $(n=296)$ were invited to complete questionnaires on chronic pain (mannequin with 18 body regions), sleeping problems (Uppsala Sleep Inventory, four items scored from 1-5), stress (ELO questions, scored from 1-5), anxiety and depression (Hospital Anxiety and Depression Scale, scored from 0-21), health status (EQ-5D, scored from 0 to 1, worst to best) and physical activity (International Physical Activity Questionnaire, categorised into low, moderate and high levels). Stress and sleeping items were dichotomized into 1-3 points (best) vs 4-5 points (worst). Individuals scoring at least severe problems (4 points) at one or more sleeping items were classified as having severe sleeping problems. HADS were categorised as non-cases $(0-7)$, possible $^{7-10}$ and probable cases (11-21 points). Students were grouped as having CMP (pain present in $\geq 3$ regions) or not (no chronic pain or chronic pain in 1-2 regions). Multiple logistic regression analyses (adjusted for sex) with CMP as dependent variable were performed in SPSS, version 24

Results: 254 students ( $86 \%$ of total sample, 87 boys and 167 girls) with a mean age of 16.1 (SD 0.6) years participated in the study. CMP was present in 25 (9.8\%) students with no differences between boys and girls $(8.0 \%$ vs $10.8 \%$; $\mathrm{p}=0.488$ ). Having CMP was associated with reporting severe sleeping problems (OR $2.49,95 \% \mathrm{Cl}: 1.06$ to $5.81, \mathrm{p}=0.035$ ) with initiating sleep, maintaining sleep, early morning awakenings and/or not feeling restored after sleep in comparison to the other students. Students with CMP were more likely to be categorised as probable cases for anxiety (OR $3.06,95 \% \mathrm{Cl}: 1.09$ to $8.61, \mathrm{p}=0.034)$, but there were no associations for possible cases for anxiety (OR $1.15,95 \% \mathrm{Cl}$ : 0.38 to 3.51 , $\mathrm{p}=0.800$ ), possible cases (OR $2.03,95 \% \mathrm{Cl}: 0.63$ to 6.54 ), or probable cases for depression (OR $3.35,95 \% \mathrm{Cl}: 0.33$ to 33.83 ). There was a nearly significant association between stress and belonging to the CMP group (OR 2.31, 95\% Cl: 0.97 to $5.53, p=0.059)$. A higher self-reported health status was associated to a lower likelihood for CMP (OR $0.04,95 \% \mathrm{Cl}: 0.01$ to $0.27, p=0.001$ ). Distribution of physical activity levels of low, moderate and high was not significantly associated to having CMP in comparison with not having it.

Conclusions: One in ten high school students fulfilled criteria for having chronic multisite musculoskeletal pain. CMP was associated to sleeping problems, anxiety, and a worse health status. The results from this study may be used by school health-care professionals in their preventive work to promote student's health.

Disclosure of Interest: None declared

DOI: 10.1136/annrheumdis-2018-eular.2917
SATURDAY, 16 JUNE 2018

\section{Stratifying connective tissue diseases}

\section{OP0362 NOVEL GENE VARIANTS ASSOCIATED WITH CARDIOVASCULAR DISEASE IN SYSTEMIC LUPUS ERYTHEMATOSUS AND RHEUMATOID ARTHRITIS}

D. Leonard ${ }^{1}$, E. Svenungsson ${ }^{2}$, J. Dahlqvist ${ }^{3}$, A. Alexsson ${ }^{1}$, L. Ärlestig ${ }^{4}$, K. E. Taylor ${ }^{5}$, J.K. Sandling ${ }^{1}$, C. Bengtsson ${ }^{4}$, M. Frodlund ${ }^{6}$, A. Jönsen ${ }^{7}$, S. Eketjäll ${ }^{8}$, K. Jensen-Urstad ${ }^{9}$, I. Gunnarsson ${ }^{2}$, C. Sjöwall ${ }^{6}$, A.A. Bengtsson ${ }^{7}$, M.-L. Eloranta ${ }^{1}$, A.-C. Syvänen ${ }^{10}$, S. Rantapää-Dahlqvist ${ }^{4}$, L.A. Criswell ${ }^{5}$, L. Rönnblom ${ }^{1} .{ }^{1}$ Dept of Medical Sciences, Science for Life Laboratory, Rheumatology, Uppsala University, Uppsala; ${ }^{2}$ Dept of Medicine, Rheumatology Unit, Karolinska Institutet, Stockholm; ${ }^{3}$ Dept of Medical Biochemistry and Microbiology, Science for Life Laboratory, Uppsala University, Uppsala; ${ }^{4}$ Dept of Public Health and Clinical Medicine/ Rheumatology, Umeå University, Umeå, Sweden; ${ }^{5}$ Rosalind Russell/Ephraim P. Engleman Rheumatology Research Center, University of California, San Francisco, San Francisco, USA; ${ }^{6}$ Dept of Clinical and Experimental Medicine, Linköping University, Linköping; ${ }^{7}$ Dept of Rheumatology, Skåne University Hospital, Lund; ${ }^{8}$ Cardiovascular and Metabolic Diseases, Innovative Medicines and Early Development Biotech Unit. AstraZeneca, Integrated Cardio Metabolic Centre (ICMC), Karolinska Institutet, Huddinge; ${ }^{9}$ Dept of Clinical Physiology, Karolinska Institutet, Stockholm; ${ }^{10}$ Dept of Medical Sciences, Science for Life Laboratory, Molecular Medicine, Uppsala University, Uppsala, Sweden

Background: Patients with Systemic Lupus Erythematosus (SLE) and Rheumatoid Arthritis (RA) have increased risk of cardiovascular disease (CVD).

Objectives: We investigated whether single nucleotide polymorphisms (SNPs) at autoimmunity risk loci were associated with CVD in SLE and RA.

Methods: SLE patients $(n=1045)$ were genotyped using the 200K Immunochip SNP array (Illumina). The allele frequency was compared between patients with and without different manifestations of CVD. Results were replicated in a second SLE cohort $(n=1043)$ and in an RA cohort $(n=824)$. We analysed publically available genetic data from the general population, performed electrophoretic mobility shift assays and measured cytokine levels and occurrence of anti-phospholipid antibodies (aPLs).

Results: We identified two new putative risk loci associated with increased risk for CVD in two SLE populations, which remained after adjustment for traditional CVD risk factors. An IL 19 risk allele was associated with stroke/myocardial infarction in SLE (OR 2.3 (1.5-3.4), $p=8.5 \times 10-5)$ and RA (OR 2.8 (1.4-5.6), $p=3.8 \times 10-$ 3 ), meta-analysis (OR $2.5(2.0-2.9), p=3.5 \times 10-7)$, but not in population controls. The $I L 19$ risk allele affected protein binding and SLE patients with the risk allele had increased levels of plasma-IL10 $(\mathrm{p}=0.004)$ and aPL $(\mathrm{p}=0.01)$. An SRP54-AS1 risk allele was associated with stroke/transient ischaemic attack in SLE (OR 1.7 (1.3-2.2), $\mathrm{p}=2.5 \times 10-5)$ but not in RA. The SRP54-AS1 risk allele is an expression quantitative trait locus for four genes.

Conclusions: The IL19 risk allele was associated with stroke/myocardial infarction in SLE and RA, but not in the general population, indicating that shared immune pathways may be involved in the CVD pathogenesis in inflammatory rheumatic diseases.

Disclosure of Interest: None declared

DOI: 10.1136/annrheumdis-2018-eular.538

OP0363

OPTIMISINGPRECISION MEDICINE BY USING GENETICS TO ASSIGN DIAGNOSTIC PRIOR PROBABILITIES TO PATIENTS WITH SYNOVITIS - PROOF OF PRINCIPLE

R. Knevel ${ }^{1,2,3}$, C. Terao ${ }^{4,5}$, J. Cui ${ }^{2}$, K. Slowikowski ${ }^{3,6}$, T. Huizinga ${ }^{1}$, B. Karlson ${ }^{2}$, K. Liao ${ }^{2}$, S. le Cessie ${ }^{7}$, S. Raychaudhuri ${ }^{3,8,9} .{ }^{1}$ Rheumatology, LUMC, Leiden, Netherlands; ${ }^{2}$ Rheumatology, Brigham and Women's Hospital, Harvard Medical School, Boston; ${ }^{3}$ Broad Institute, Cambridge, USA; ${ }^{4}$ Rheumatology, University of Shizuoka, Shizuoka: ${ }^{5}$ RIKEN Center for Integrative Medical Sciences, Kanagawa, Japan; ${ }^{6}$ Genetics, Brigham and Women's Hospital, Harvard Medical School, Boston, USA; ${ }^{7}$ Epidemiology, LUMC, Leiden, Netherlands; ${ }^{8}$ Rheumatology/ Genetics, Brigham and Women's Hospital, Harvard Medical School, Boston; ${ }^{9}$ Rheumatology, university of Manchester, Manchester, USA

Background: In patients with synovitis, the question is 'Which disease does this patient have?' However, traditional tests often only inform us about disease presence yes/no and disease discriminating symptoms often take a while to arise. Time independent information, such as genetics, might accelerate the diagnostic process. As increasing number of patients have genotyping data available in medical records prior to their visit, the question emerges: can genetic data facilitate disease differentiation in early disease?

Objectives: Proof of principle study to test the differentiating ability of genetic profiles in patients with synovitis.

Methods: We studied the most common rheumatologic diseases: rheumatoid and psoriatic arthritis, SLE, spondyloarthropathy and Gout. The population level 